\title{
Microcooling Developments at University of Twente
}

S. Vanapalli, P. Lerou

University of Twente,

The Netherlands

MICMEC

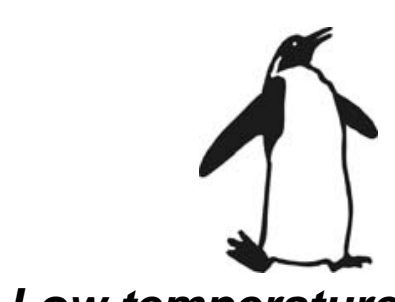

Low temperature division 
Cooling needs in Space

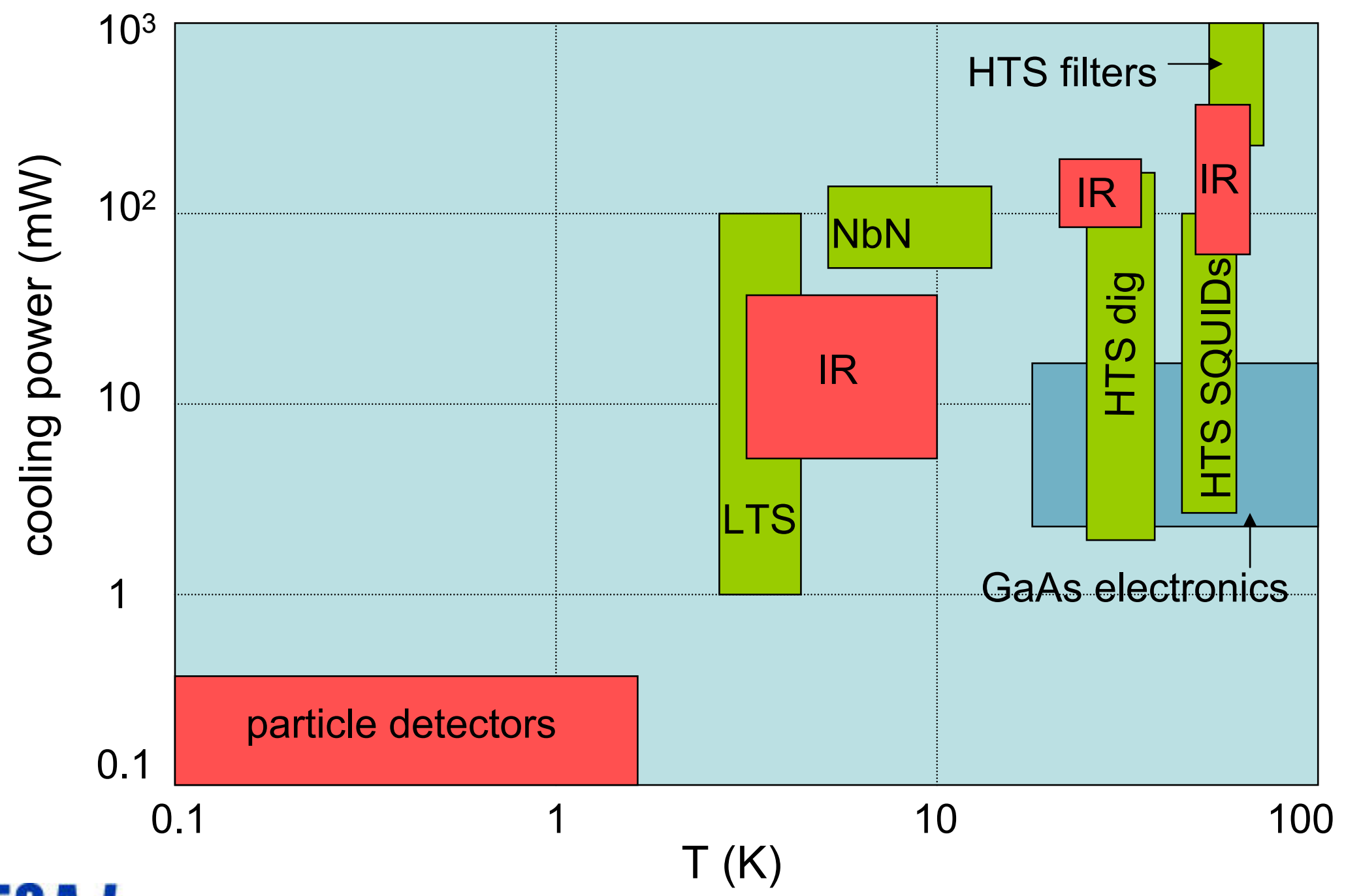




\section{Cooling options in Space}

mass per unit of cooling power $(\mathrm{kg} / \mathrm{mW})$

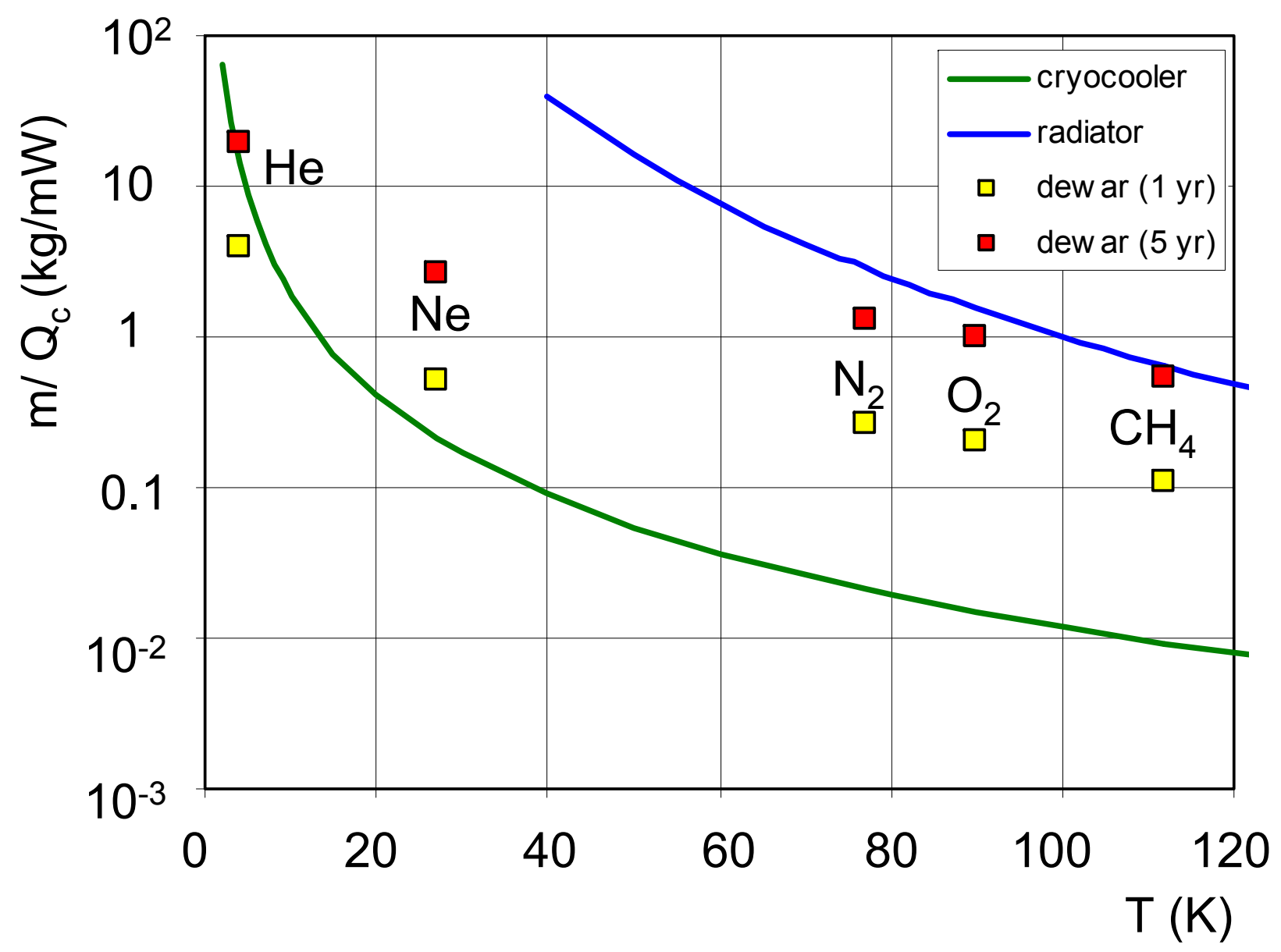

$\longrightarrow$ lower T means larger cooling system

$\longrightarrow$ cryocoolers attractive because of size and weight 

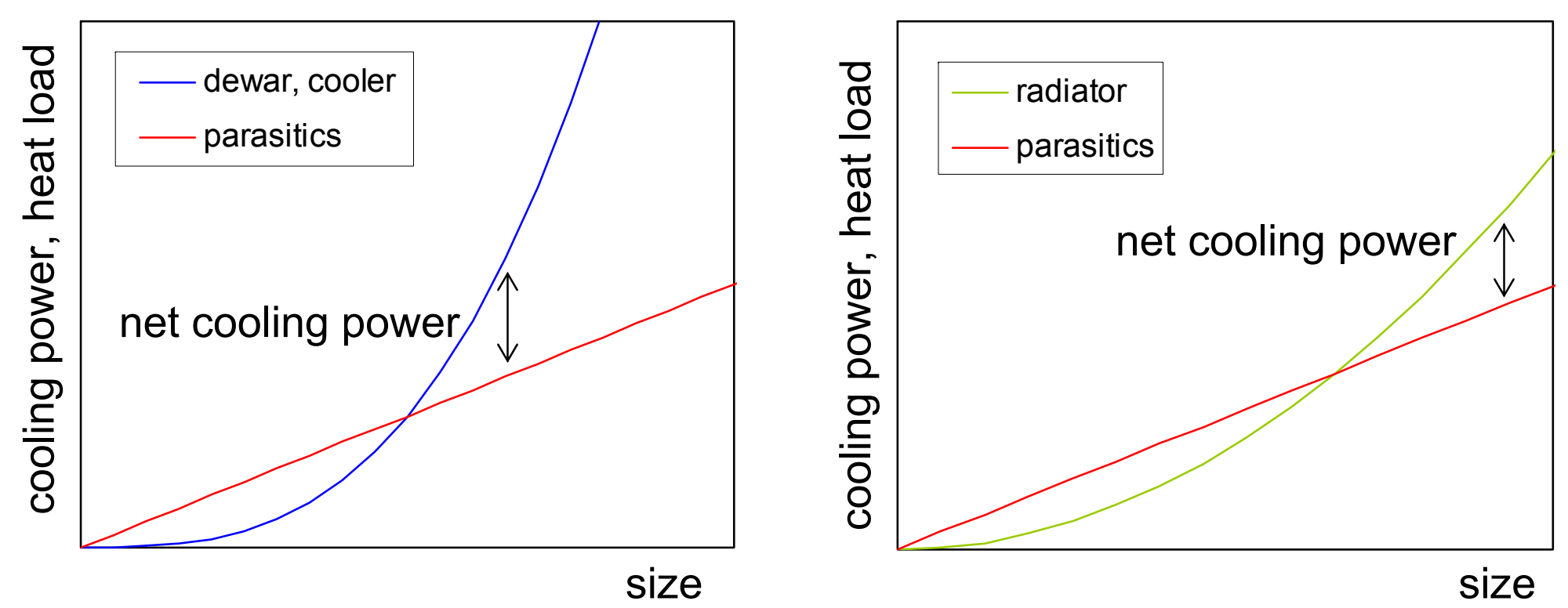

In cryocooler design the dependence of cooling power on size can be influenced

regenerative cooler: $\quad \dot{Q}_{L} \sim f \times \Delta p \times V_{\text {gas }} \longrightarrow$ frequency $f$ can be increased recuperative cooler: $\dot{Q}_{L}=\dot{m} \times \Delta H_{\text {gas }} \longrightarrow$ compressor design can be used with size-independent flow (e.g. sorption) 


\section{Cooling via expansion of compressed gas}

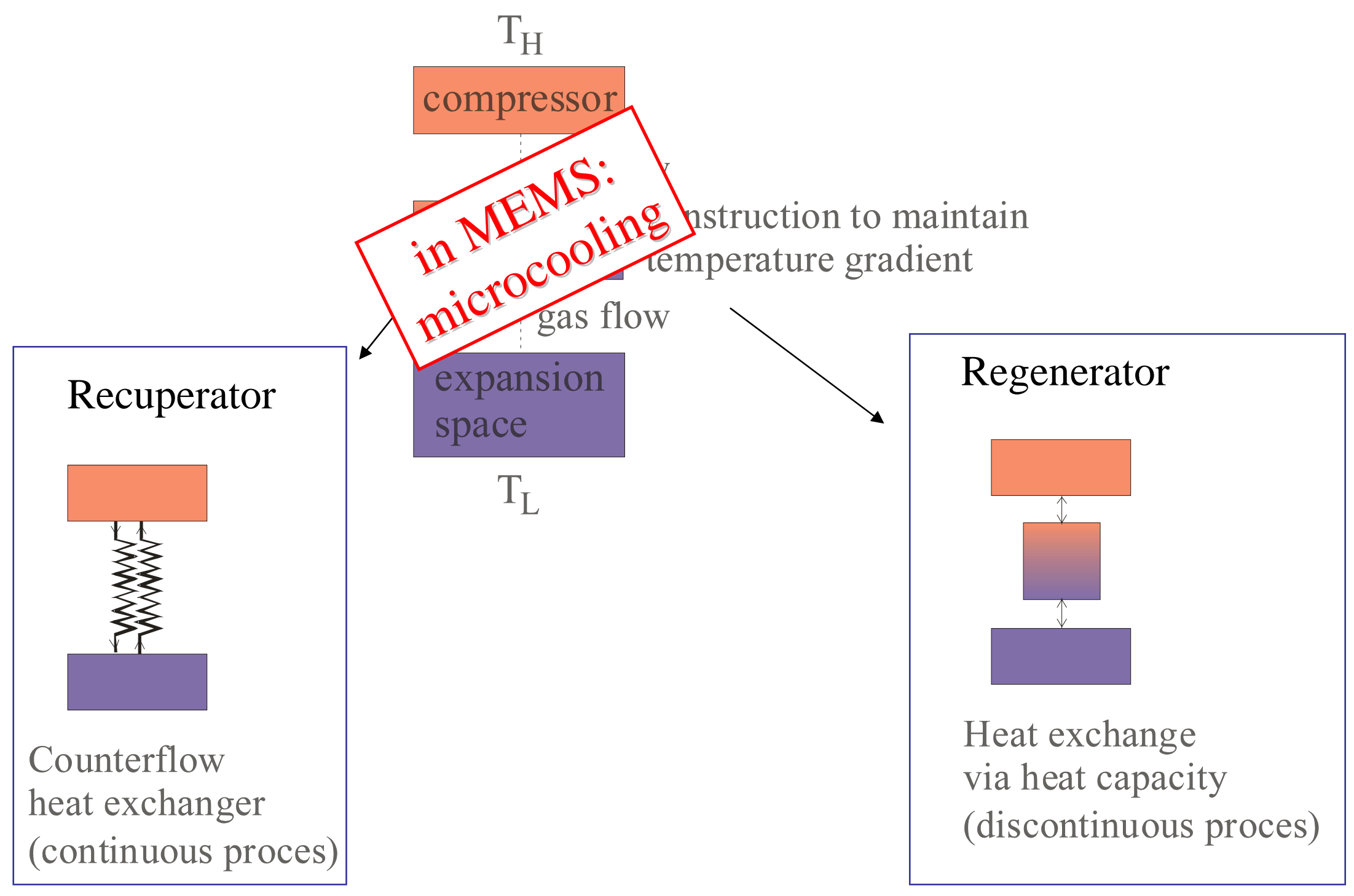




\section{Contents:}

Microcooling research in Twente

micro-recuperative cooler

micro-regenerative cooler 


\section{Recuperative Cooler}

\section{JT Cycle:}

- Gas: $\mathrm{N}_{2}$

- $\mathrm{P}_{\text {high }}=80 \mathrm{bar}$

- $\mathrm{P}_{\text {low }}=6$ bar

- $\mathrm{T}_{\text {cold stage }}=96 \mathrm{~K}$

- $\mathrm{mflow}=1 \mathrm{mg} / \mathrm{s}$

- $\mathrm{P}_{\text {gross }} \approx 14 \mathrm{~mW}$

- $\mathrm{P}_{\mathrm{net}} \approx 10 \mathrm{~mW}$
Low pressure line

High pressure line

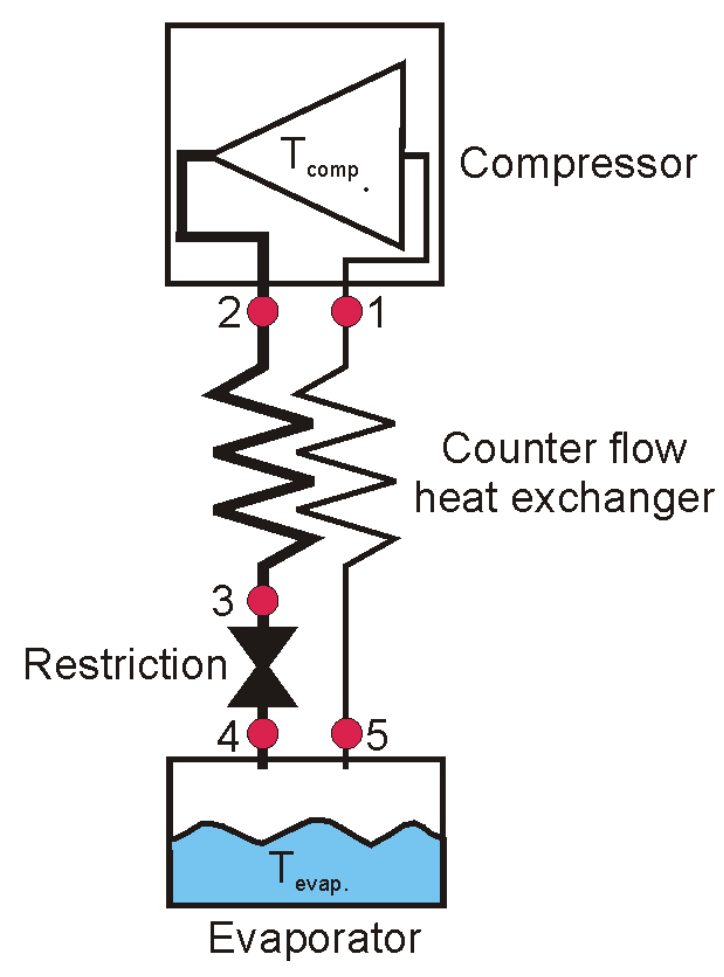


Cold-tip prototype

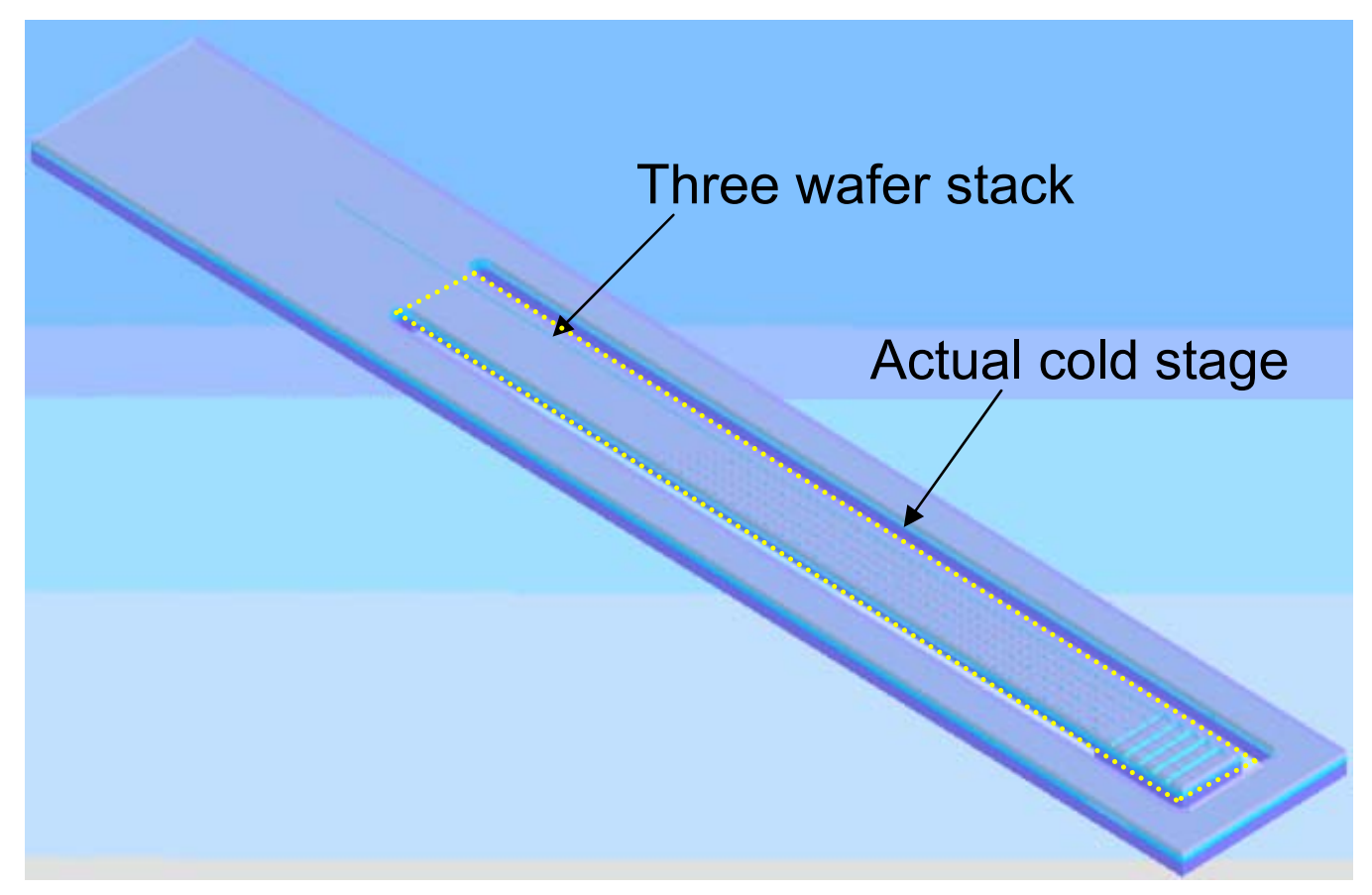

cold-stage length: $28 \mathrm{~mm}$

cold-stage width: $2.2 \mathrm{~mm}$ 


\section{Cold-tip prototype}

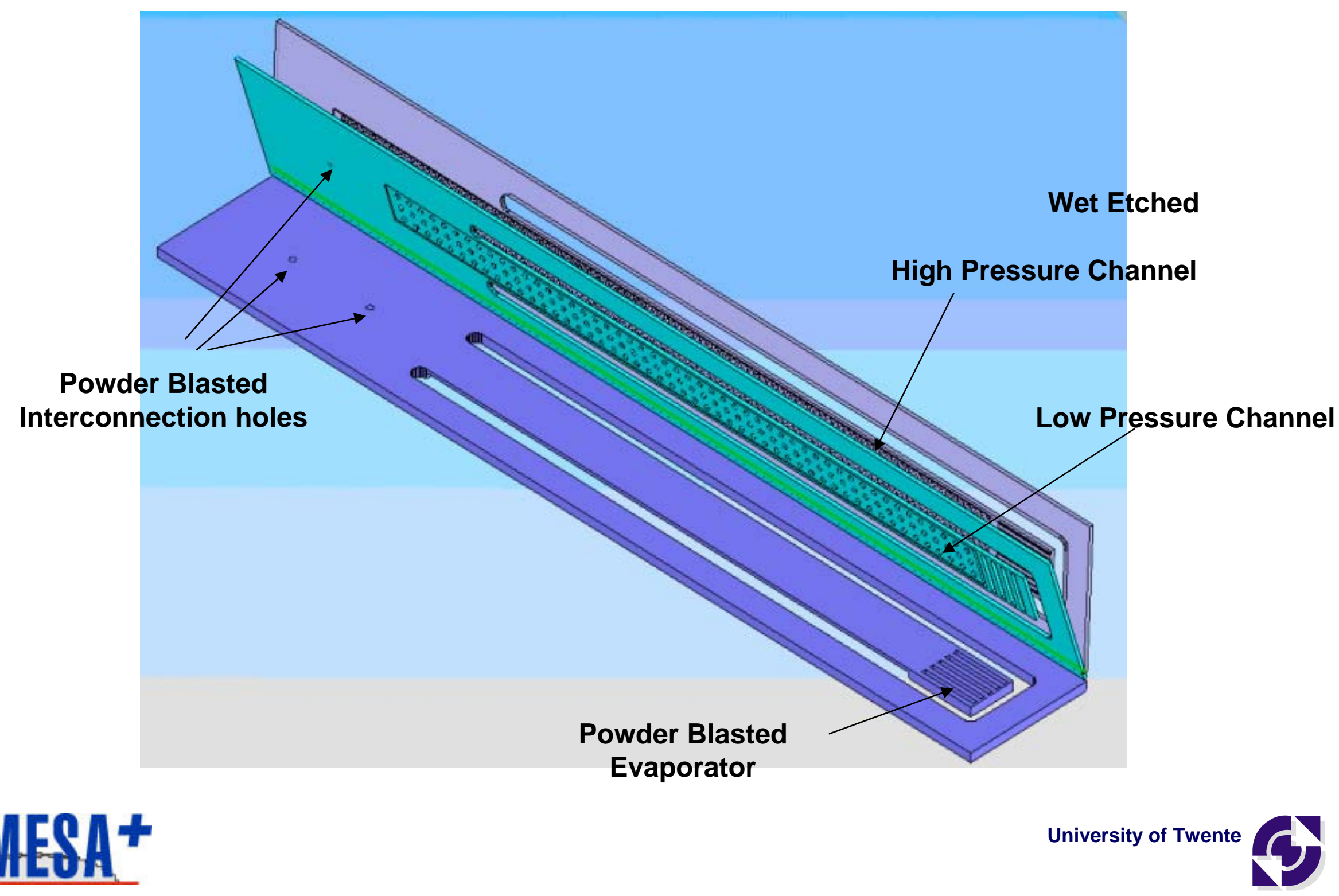




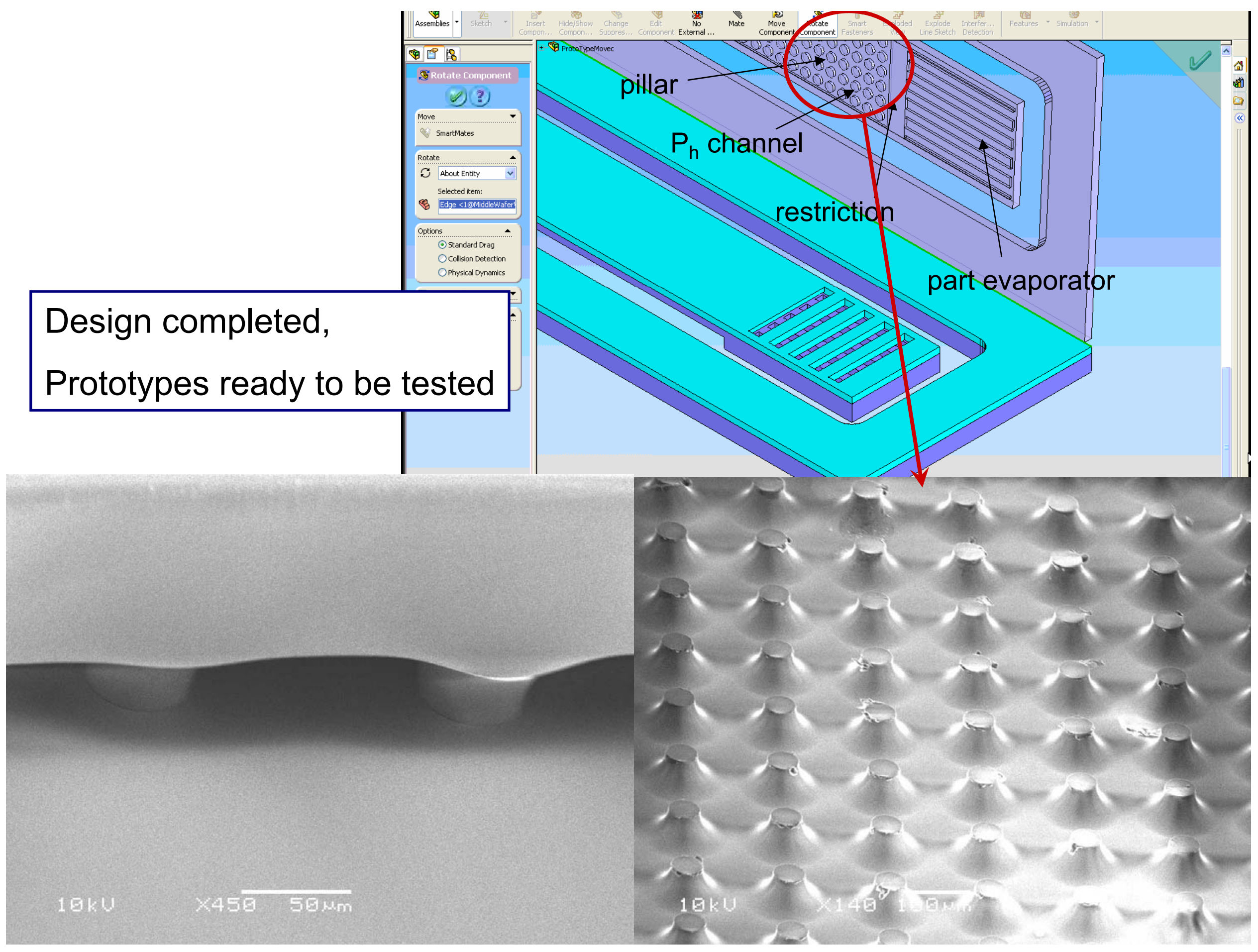




\section{Micro vacuum chamber}

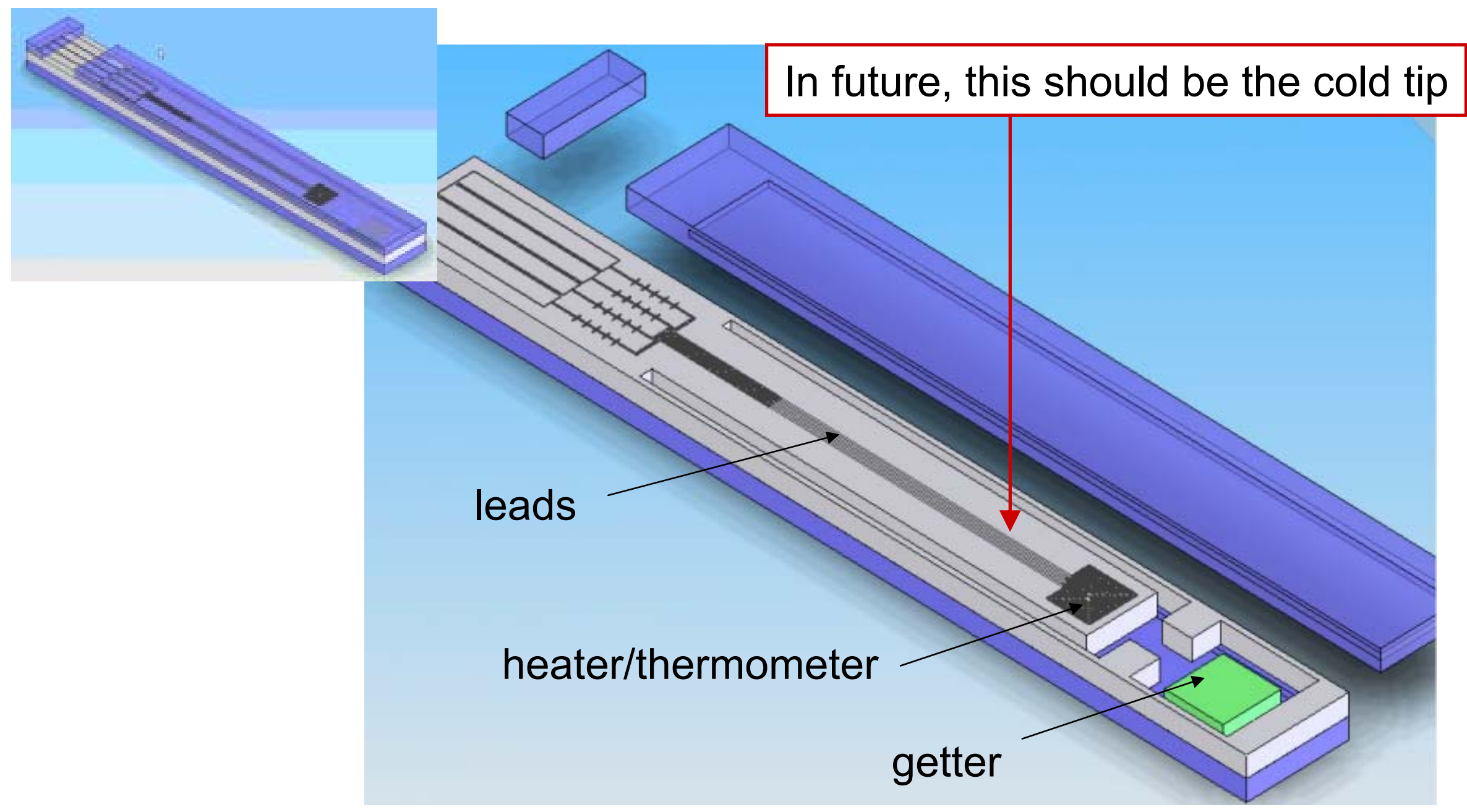

- test chamber under construction: gas pressure to be monitored via thermal conductivity (Pirani-principle) 


\section{Micro-regenerative cooling:}
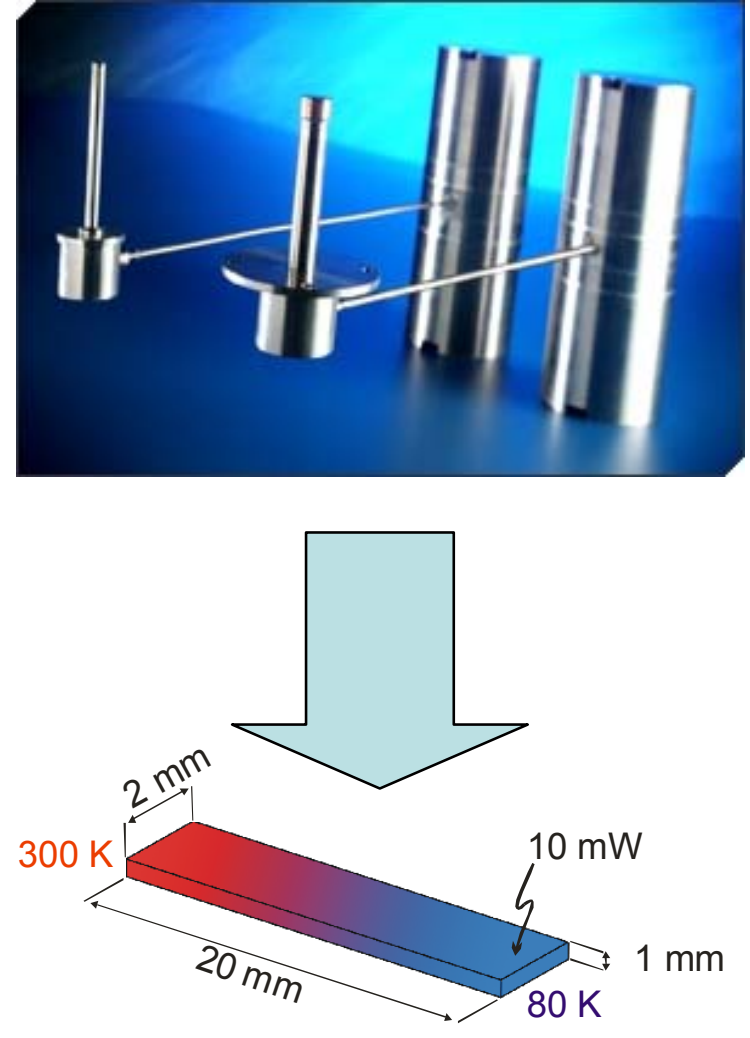

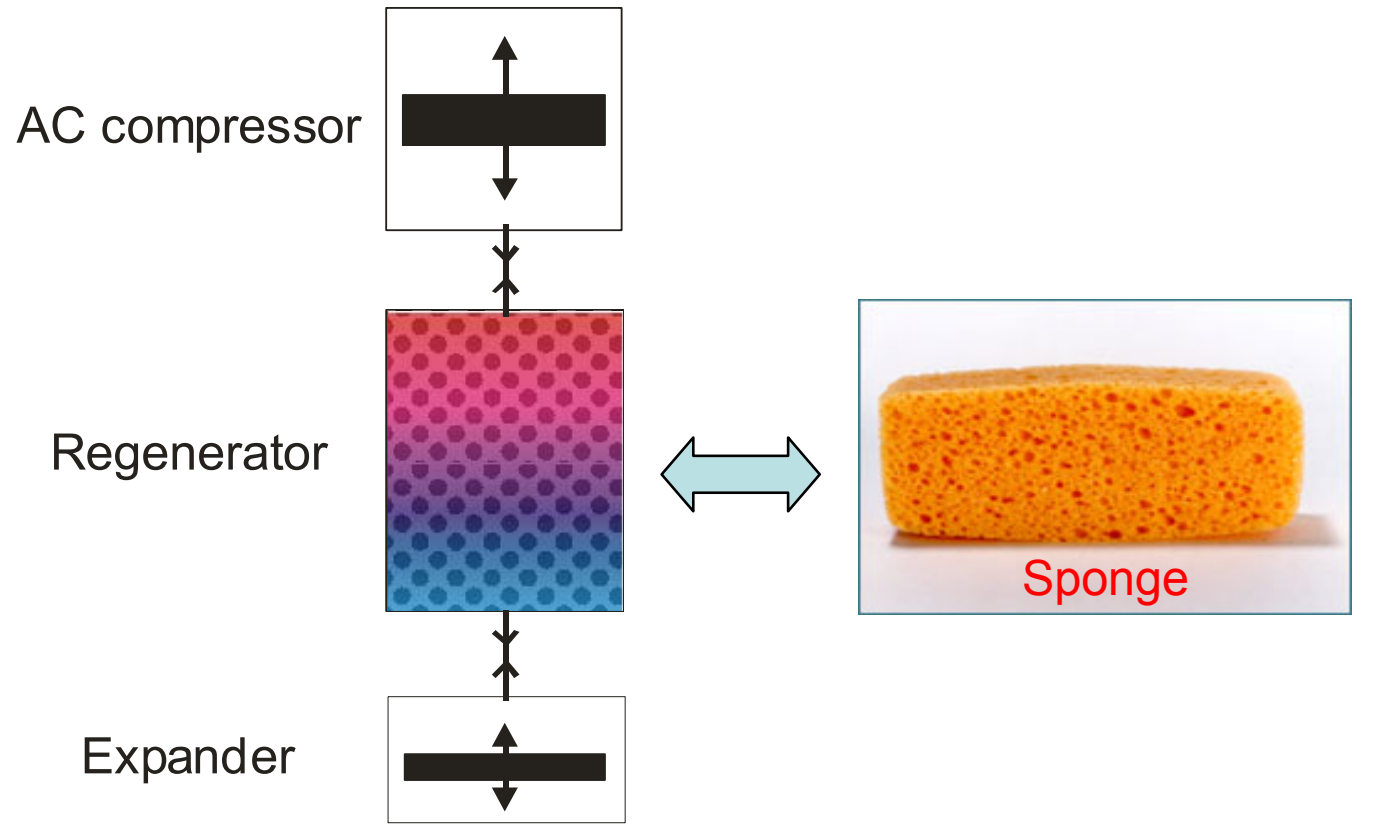

Regenerator exchanges heat alternately with gas 


\section{Regenerator is critical element}

\section{Example:}

\section{Cooling Power 30 mW @ 100K}

Stored Heat flux in Regenerator

$$
Q_{\text {reg }}=m C_{p, g}\left(T_{\text {amb }}-T_{\text {cold }}\right) \approx \mathbf{4 0 0} \mathbf{m W}
$$

If we accept loss of $\mathbf{2} \%, \rightarrow \mathbf{8} \mathbf{~} \mathbf{W}$ lost from cooling power

Optimization of Regenerator is critical!! 
MEMS enables regenerator matrix optimization
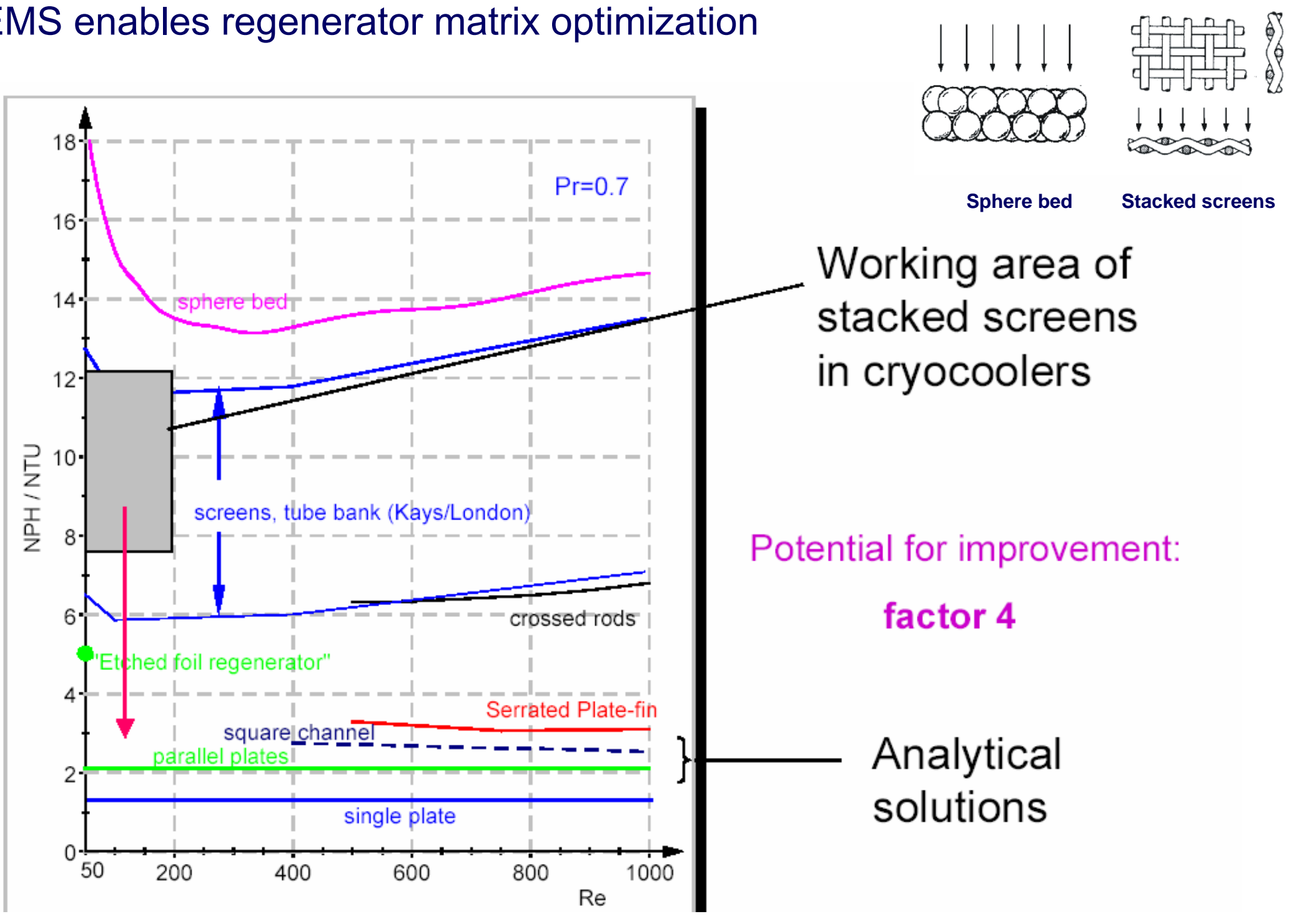

Working area of stacked screens

in cryocoolers

Potential for improvement:

factor 4

Analytical

solutions 


\section{Fabrication}

Regenerator Pillar Matrix - Optimized Bosch Process

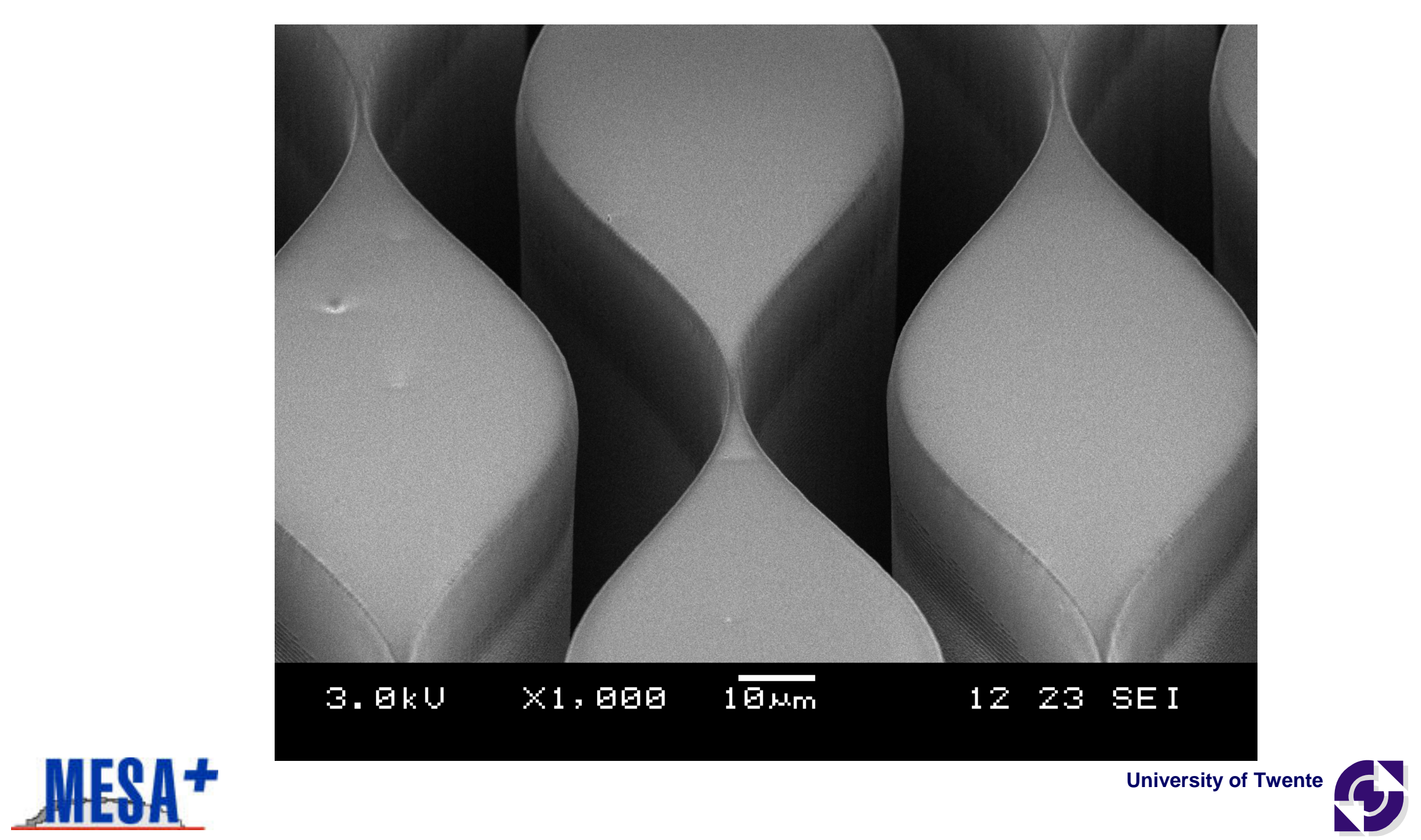




\section{Pressure drop measurements}

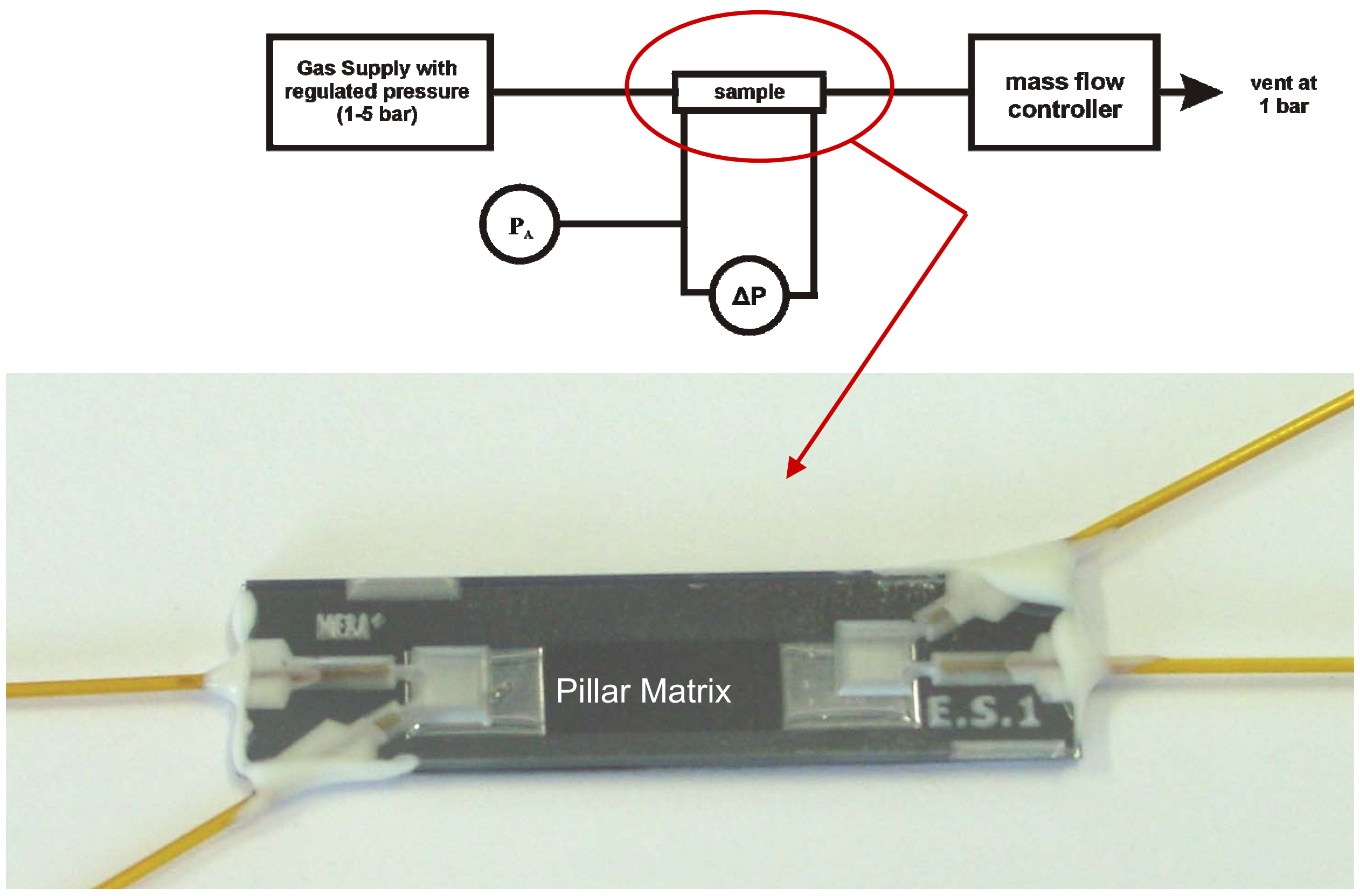




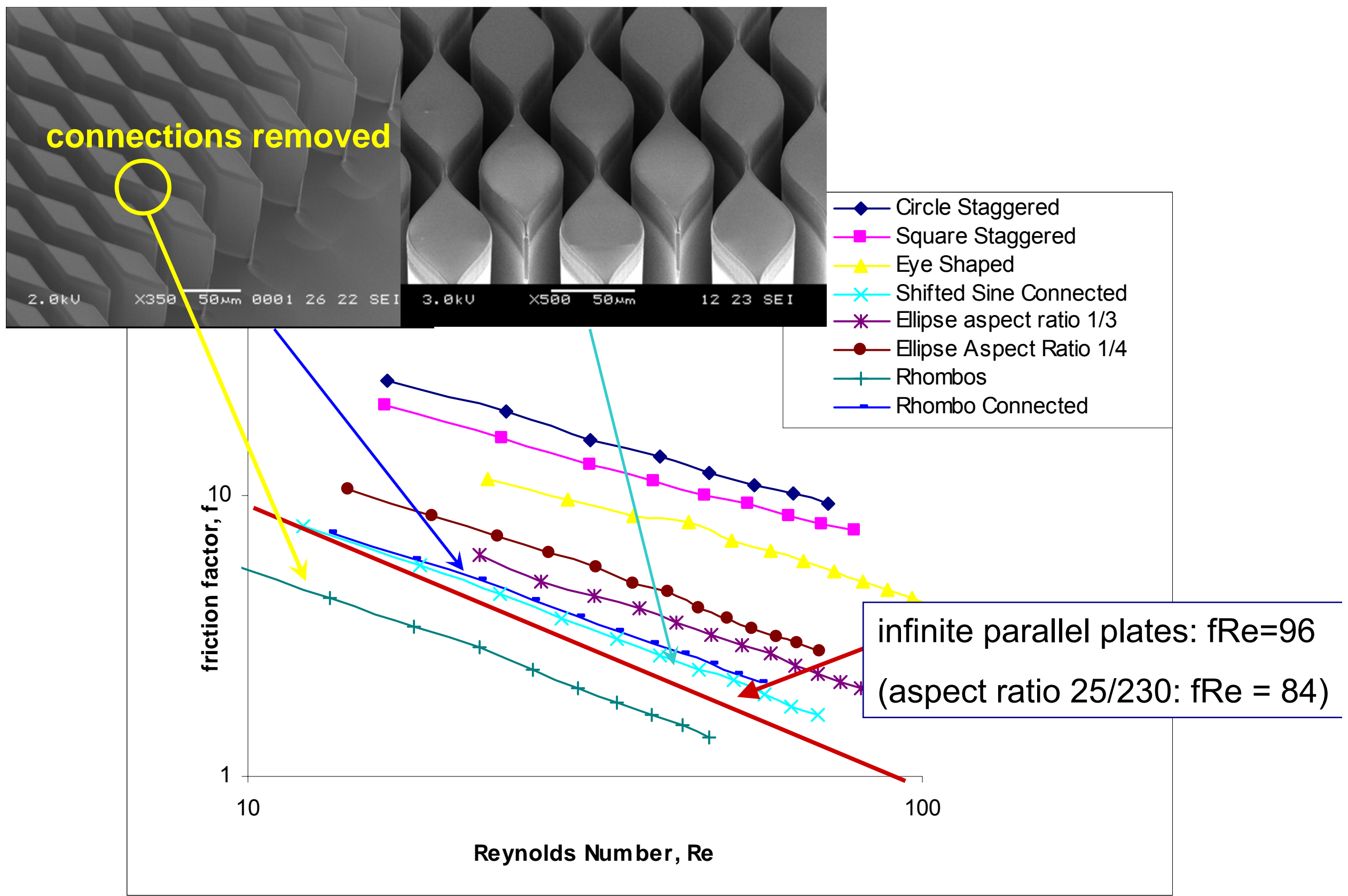




\section{Status - Outlook}

- Micro cryocooler has high potential for space applications

- The total volume of the cold stage $<<1 \mathrm{~cm}^{3}, 10 \mathrm{~mW} @ 96 \mathrm{~K}$

- Total integration of cold stage, device, vacuum chamber and compressor

Micro-recuperative cooling

- Optimal design of counter flow heat exchanger is complete,

- First prototypes are ready to be tested.

- Multi staging: 5 mW @ $27 \mathrm{~K}$

Micro-regenerative cooling

- Various micro regenerator geometries were fabricated.

- Hydraulic and Thermal characterization of different geometries is done. 


\section{Acknowledgements}

Prof. Marcel ter Brake

Prof. Miko Elwenspoek

Prof. H. Rogalla

Henri Jansen

Johannes Burger

Theo Veenstra

Gunter Venhorst
Joost Koning

Yiping Zhao

Berker Mogulkoc 